\title{
Bioinformation Analysis Reveals IFITI as Potential Biomarkers in Central Nervous System Tuberculosis
}

\author{
Wenbin Qiaol,* \\ Jiahui Fan $^{1, *}$ \\ Xiaoqian Shang ${ }^{l} *$ \\ Liang Wang ${ }^{2}$ \\ Bahetibieke Tuohetaerbaike ${ }^{2}$ \\ Ying $\mathrm{Li}^{2}$ \\ Li Zhang ${ }^{2}$ \\ YiShan Huo' \\ Jing Wang ${ }^{3}$ \\ Xiumin $\mathrm{Ma} \mathbb{D}^{1,2}$ \\ 'State Key Laboratory of Pathogenesis, \\ Prevention and Treatment of High Incidence \\ Diseases in Central Asia, Clinical Laboratory \\ Center, Tumor Hospital Affiliated to \\ Xinjiang Medical University, Urumqi, \\ 830000, People's Republic of China; ${ }^{2}$ First \\ Affiliated Hospital of Xinjiang Medical \\ University, Urumqi, Xinjiang, 8300 I I, \\ People's Republic of China; ${ }^{3}$ Respiratory \\ Department of the Second Affiliated \\ Hospital of Hainan Medical College, Haikou, \\ Hainan, 570000, People's Republic of China \\ *These authors contributed equally to this \\ work
}

Correspondence: Xiumin Ma State Key Laboratory of Pathogenesis, Prevention and Treatment of High Incidence Diseases in Central Asia, Clinical Laboratory Center, Tumor Hospital Affiliated to Xinjiang Medical University, No. 789 Suzhou Road, Urumqi, 8300I I, Xinjiang Uygur

Autonomous Region, People's Republic of China

Tel +86-13369649029

Email maxiumin1210@sohu.com

Jing Wang

Respiratory Department of the Second

Affiliated Hospital of Hainan Medical

College, Haikou, Hainan, 570000, People's

Republic of China

Tel +86-13629925886

Email tlfwj@I63.com
Objective: Central nervous system tuberculosis is the most serious form of extrapulmonary tuberculosis. We aim to discover potential biomarkers involved in the development of the disease. Methods: Through gene difference analysis, construction of a protein interaction network and tissue specific analysis and other bioinformatics analysis methods, we found out the relatively high expression of important substances in the central nervous system, interferon induced protein with tetratricopeptide repeats 1 . Subsequently, the lesion tissue and the resection margin tissue away from the lesion were collected from the 6 cases of central nervous system tuberculosis patients diagnosed from 2019 to 2020, and the pathological manifestations were observed by Hematoxylin and Eosin (H\&E) staining, and the expression of IFIT1 was verified by immunohistochemistry.

Results: A total of 101 differential genes were analyzed between extrapulmonary tuberculosis patients and normal people, and they were mainly enriched in the interferon pathway. The protein interaction network unearthed 34 key genes. Through tissue specific analysis, it was found that IFIT1 is relatively high in the central nervous system. H\&E staining showed the expression of multinucleated macrophages, and immunohistochemistry showed that IFIT1 was significantly positively expressed in the lesion tissue.

Conclusion: IFIT1 is an important substance involved in central nervous system tuberculosis.

Keywords: central nervous system tuberculosis, biomarkers, interferon induced protein with tetratricopeptide repeats 1 , hematoxylin and eosin staining, immunohistochemistry staining

\section{Introduction}

Tuberculosis (TB) is considered to be one of the top ten causes of death in the world, about a quarter of the world's population is infected with M. tuberculosis. ${ }^{1}$ The World Health Organization (WHO) divides tuberculosis into pulmonary tuberculosis (PTB) and extra-pulmonary tuberculosis (EPTB). Although breakthroughs have been made in the prevention and treatment of PTB, the incidence of EPTB has reached $16 \%,{ }^{3}$ and the available epidemiological information shows, the incidence of EPTB is gradually increasing in China. ${ }^{2,3}$ In fact, due to non-specific symptoms, chronic, hidden and changeable clinical manifestations, clinicians' low awareness of EPTB, and lack of accurate tools to detect the extrapulmonary diseases, the incidence of EPTB is not accurately estimated globally. ${ }^{4-6}$ Central nervous system tuberculosis (CNS-TB) is one of the most destructive forms of extrapulmonary tuberculosis, and is the second most common community-acquired CNS infection worldwide. Its unacceptable case fatality rate and mortality rate have attracted the wide attention of scholars. ${ }^{7,8}$ 
CNS-TB is a severe form of EPTB. The incidence of clinical CNS-TB accounts for $1-2 \%$ of all active TB patients, and accounts for about $8 \%$ of extrapulmonary infection cases. ${ }^{9}$ Despite the effective anti-TB chemotherapy, the fatality rate $(15-40 \%)$ is still high. ${ }^{9,10}$ CNS-TB is mainly characterized by tuberculous meningitis (TBM), is less manifested as tubercular encephalitis, intracranial tuberculoma or tuberculous brain abscess. They are usually caused by hematogenous transmission of primary pulmonary infection with mycobacterium tuberculosis (M. tb). In the spore stage, sparse bacillus can spread throughout the brain tissue, meninges and adjacent tissues, forming multiple granulomatous foci tubercles with different sizes and different degrees of encapsulation, continuous growth and merging of tubercles lead to larger caseous lesions. ${ }^{10}$ A distinctive feature of $\mathrm{M}$. tb is that it can enter macrophages and be in stock. In the central nervous system, microglia are resident macrophages, therefore, human microglia are major targets of M. tb infection in the central nervous system. ${ }^{11}$ Microglia-related cytokines, such as IFN, TNF, interleukin and chemokines, have been extensively studied. ${ }^{12-14}$ However, these cytokines are also important markers of other types of EPTB, there is a lack of specificity for CNS-TB. We hope to explore potential molecular markers involved in the development of CNS-TB through bioinformatics analysis.

We downloaded the Blankley et a ${ }^{15}$ created microarray dataset GSE83456 from the public database (Gene Expression Omnibus database, GEO), used bioinformatic methods to compare and analyze the original genetic data of the whole blood of the patients with extrapulmonary TB and healthy people, We searched for the key genes by constructing the protein-protein interaction (PPI) network map, and then, through tissue specificity analysis, potentially important biomarkers involved in the occurrence and development of CNS-TB were excavated, immunohistochemical (IHC) staining was used to verify. Our results are helpful to reveal the pathogenesis of CNS-TB at the molecular level.

\section{Materials and Methods}

\section{Original Data Acquisition}

"Tuberculosis" was retrieved as a keyword on the GEO (http://www.ncbi.nlm.nih.gov/geo) public database, We downloaded the microarray expression profiling dataset GSE83456 that Blankley et $\mathrm{al}^{15}$ saved in 2016. The data set was based on the GPL10558 Illumina microarray platform HumanHT-12 v4.0 Expression BeadChip, and collected 47 cases of EPTB and 61 cases of healthy control (HC). Active-tuberculosis meets one of the following conditions: (1) Positive mycobacterial culture result from the site of disease. (2) Caseating granuloma on biopsy and/or clinical/radiological features consistent with active TB and a good response to therapy. Since the dataset and annotation files were downloaded from public databases, patient consent and ethics committee approval are not required.

\section{Data Process}

RStudio software (v4.0.3) was used to standardize data preprocessing and ID conversion. The FactoMineR package was used to perform principal component analysis (PCA) and draw the diagram. Differentially expressed genes between EPTB and HC were screened by the limma package. ${ }^{16}$ Adjusted $\mathrm{p}$ value of $<0.05$ and $\mid \log 2$ (fold-change) $\mid>1$ were considered significant and qualified genes were included in a differentially expressed genes set (DEGs) and we used a ggplot2 package and Pheatmap package to draw figures.

\section{DEGs Enrichment Analysis}

Metascape (http://metascape.org/gp/index.html) online database updates over 40 knowledge bases in its platform monthly, in order to provide the most accurate results. Therefore, DEGs was entered into it for Gene Ontology (GO) and Kyoto Encyclopedia of Genes and Genomes (KEGG) pathway enrichment analysis. ${ }^{17}$ To further verify the enrichment pathway of gene expression, all gene sets were uploaded to GSEA software (v4.1.0) for more detailed analysis. Normalized enrichment score (NES) $>2$, false discovery rate (FDR) $<0.05$ and normal $\mathrm{P}$ value $<0.01$, considered statistically significant.

\section{PPI Network Creation and Hub Gene Identification}

In order to continue to understand the interaction between different genes, online STRING database (https://stringdb.org/) and Cytoscape software (v3.8.0) were used to display PPI diagrams and screen hub genes. ${ }^{18,19}$ The DEGs was entered into online STRING and set an interaction score $>0.7$ as statistically significant. An interaction network of component-target was visualized by Cytoscape software. In addition, for identifying key gene modules, the app MCODE (v2.0.0), a plug-in of Cytoscape software, was used to identify closely connected network 
clusters, the scoring parameters of cluster analysis followed the default parameters of the app.

\section{Tissue-Specific Gene Expression Analysis}

The online resource BioGPS (http://biogps.org) gene expression profile was used to identify the specific distribution of genes in tissues and organs. In order to screen the important target genes involved in CNS-TB and obtain more biological information of important genes, the important genes identified by MCODE were uploaded to the website for tissue-specific expression analysis. It was considered that the data set was the result of whole blood sequencing, so the expression of genes in whole blood was excluded. In other tissues or organs, genes meeting the following conditions is considered to be highly expressed:

(1) The tissue-specific expression level was $>5$ times the median. (2) The second highest expression level was below $1 / 3$ of the highest level. ${ }^{20}$

\section{Clinical Sample Collection}

The sample group contained 6 patients with CNS-TB, and 6 patients with PTB. They underwent surgery in the first affiliated Hospital of Xinjiang Medical University between January 2019 to December 2020, we collected the lesion tissue and tissue far from the focus site. The clinical medical records of the $6 \mathrm{CNS}-\mathrm{TB}$ patients were collected. Inclusion criteria were: (1) The etiological culture of the focus was positive. (2) Or the biopsy and/or clinical/radiological features were consistent with TB, and there was a good response to antituberculosis drug therapy. Exclusion criteria were: (1) Patients with other lung diseases. (2) Patients with autoimmune diseases. ${ }^{15}$ All patients had signed the informed consent for the study protocol, and the study was approved by the Local Ethics Committee of Xinjiang Medical University. Our research obeyed the Declaration of Helsinki.

\section{Hematoxylin and Eosin (H\&E) Staining}

Paraffin sections were deparaffinized with xylene and ethanol, stained with hematoxylin-eosin, dehydrated with ethanol, made transparent with xylene, and sealed with neutral gum. Finally, CNS-TB lesions were observed under a microscope and images were collected.

\section{Immunohistochemistry (IHC) Staining}

After dewaxing, the tissue sections were repaired with sodium citrate solution for 15 minutes, the endogenous peroxidase blocker was placed away from light at $37^{\circ} \mathrm{C}$ for 10 minutes, the goat serum was sealed for 20 minutes, and the anti-IFIT1
(1:200, Bioss China) was incubated overnight in a refrigerator at $4{ }^{\circ} \mathrm{C}$. The next day, the second antibody was combined for 90 minutes at $37^{\circ} \mathrm{C}$. The staining was performed with diaminobenzidine in peroxide substrate solution for $2.5 \mathrm{~min}$. The results of IHC were analyzed by Image-Pro Plus Version 8.0.1, after quantitative processing (IOD (sum)/Area (sum)), the positive rate was calculated.

\section{Statistical Analysis}

GraphPad Prism 8.0.1 was used for calculation and visualization. For data that do not obey normal distribution, the differences between variables were analyzed by the Wilcoxon test method, $\mathrm{p}$ value $<0.05$ was considered statistically significant.

\section{Results \\ Identification of DEGs}

PCA maps and heat maps were drawn to understand whether there were differences in microarray matrices (Figure 1A and B). It could be seen that there were significant differences in gene expression between EPTB and HC. Subsequently, the differential genes were screened out by RStudio software, and the conditions: adjusted $\mathrm{p}$ value $<0.05$ and $|\log 2 \mathrm{FC}|>1$ (Table 1 ), and then draw a volcano map (Figure 1C). Therefore, DEGs has a total of 101 differential genes, including 91 upregulated genes and 10 down-regulated genes.

\section{Signaling Pathway Enrichment in DEGs}

DEGs was uploaded to the Metascape online database, and KEGG Pathway, GO Biological Processes, Transcription Factor Targets and COVID were used for enrichment analysis, with a default $p$ value $<0.01$, a minimum count of 3 and an enrichment factor $>1.5$. The top 20 significant enrichment pathways are shown here (Figure 1D). As shown in Figure 1D, DEGs was significantly enriched in the interferon pathway. Subsequently, the microarray data of all gene expressions were submitted to GSEA software, h.all.v 6.2. symbols.gmt [Hallmarks] gene set database was used to process data, normalized $\mathrm{p}$ value $<0.01$, false discovery rate (FDR) q value $<0.05$ and enrichment score $>2$ were considered as significant and were displayed (Figure 2, Table 2). The EPTB gene set was significantly enriched in the inflammatory response pathway, IFN response pathway, NF- $\kappa \mathrm{B}$ mediated TNF signal pathway and complement signal pathway. Therefore, extrapulmonary tuberculosis is mainly an inflammatory reaction, and immune-related cytokines such 

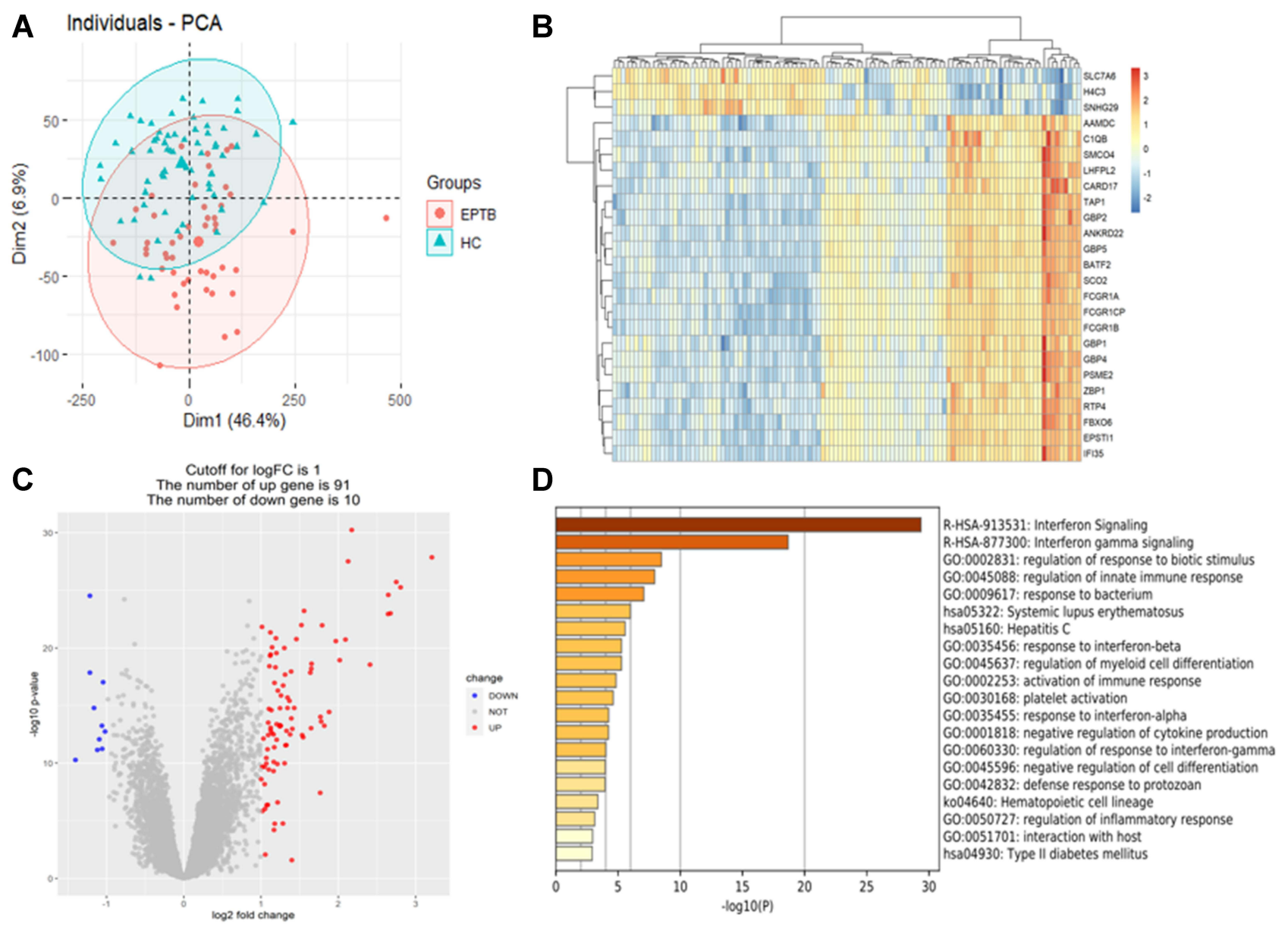

Figure I Difference analysis and signaling pathway enrichment. (A) Principal component analysis diagram, red dots for extra-pulmonary tuberculosis, blue triangles for healthy control. (B) Heatmap was drawn for the top 25 differentially expressed genes set. (C) Differentially expressed genes set drew a volcanic map, 91 red dots represented up-regulated genes and 10 blue dots represented down-regulated genes. (D) Metascape analyzed of the first 20 significant enrichment pathways of differentially expressed genes set.

as IFN- $\gamma$, IFN- $\alpha$ and TNF play an important role in the occurrence and development of the disease.

\section{PPI Network and Tissue-Specific Expression}

The DEGs were submitted to the STRING online database, and a PPI network consisting of 66 nodes and 509 edges was drawn. The results were visualized through Cytoscape software, and then the MCODE app was used to screen out 3 main gene clusters, a total of 34 key genes (Figure 3A, Table 3). These key genes were all up-regulated genes and were used in the next step of tissue-specific analysis.

34 key genes were analyzed by the BioGPS website for expression in different tissues and organs (Table 4). Remarkably, we were fortunate to screen for the gene with a high expression in the central nervous system, interferon induced protein with tetratricopeptide repeats 1 (IFIT1). This gene belonged to the first gene cluster selected by MCODE, so we used Cytoscape software to draw and display it again (Figure 3B).

\section{Clinical Data Analysis}

The laboratory examination data of 6 CNS-TB patients is shown in Table 5. 6 patients had no history of tuberculosis and were not treated with anti-tuberculosis drugs. Most of the patients had obvious dizziness and headache symptoms, and two patients had vomiting symptoms. Radiographic examinations mainly indicate space-occupying lesions, in addition, pathological diagnosis indicates typical TB pathological manifestations such as tuberculous granuloma or caseous necrosis.

\section{H\&E Staining}

After HE staining, it could be seen under the microscope that there were typical Langham's multinucleated macrophages and large pieces of caseous necrosis in the CNS- 
Table I Differentially Expressed Genes

\begin{tabular}{|c|c|c|c|}
\hline Gene Symbol & Adjusted $P$-value & Fold Change & Gene Title \\
\hline \multicolumn{4}{|c|}{ Up-regulated genes } \\
\hline BATF2 & I.44E-24 & 3.21 & Basic leucine zipper ATF-like transcription factor 2 \\
\hline ANKRD22 & $2.30 \mathrm{E}-22$ & 2.80 & Ankyrin repeat domain 22 \\
\hline FCGRICP & $9.90 \mathrm{E}-23$ & 2.75 & Fc fragment of IgG receptor Ic, pseudogene \\
\hline FCGRIA & I.90E-20 & 2.67 & Fc fragment of lgG receptor la \\
\hline FCGRIB & $8.55 \mathrm{E}-22$ & 2.65 & Fc fragment of lgG receptor $\mathrm{lb}$ \\
\hline CIQB & I.97E-20 & 2.64 & Complement $\mathrm{Clq} \mathrm{B}$ chain \\
\hline RSAD2 & I.47E-16 & 2.41 & Radical S-adenosyl methionine domain containing 2 \\
\hline GBP5 & $1.20 \mathrm{E}-26$ & 2.17 & Guanylate binding protein 5 \\
\hline EPSTII & 2.17E-24 & 2.13 & Epithelial stromal interaction I \\
\hline CARDI7 & I.84E-18 & 2.09 & Caspase recruitment domain family member 17 \\
\hline IFIT3 & 7.IIE-I7 & 2.02 & Interferon induced protein with tetratricopeptide repeats 3 \\
\hline GBPI & $2.39 \mathrm{E}-18$ & 1.97 & Guanylate binding protein I \\
\hline ISGI5 & $6.50 \mathrm{E}-13$ & 1.88 & ISGI5 ubiquitin like modifier \\
\hline IDOI & $6.95 \mathrm{E}-12$ & 1.82 & Indoleamine 2,3-dioxygenase I \\
\hline RTP4 & $1.65 \mathrm{E}-19$ & 1.79 & Receptor transporter protein 4 \\
\hline CXCLIO & $3.30 \mathrm{E}-12$ & 1.78 & $\mathrm{C}-\mathrm{X}-\mathrm{C}$ motif chemokine ligand $\mathrm{IO}$ \\
\hline CASP5 & $1.48 \mathrm{E}-12$ & 1.77 & Caspase 5 \\
\hline IFI27 & $8.99 \mathrm{E}-07$ & 1.76 & Interferon alpha inducible protein 27 \\
\hline CEACAMI & $1.24 \mathrm{E}-16$ & 1.65 & CEA cell adhesion molecule I \\
\hline ETV7 & $3.06 \mathrm{E}-16$ & 1.65 & ETS variant transcription factor 7 \\
\hline IFITI & I.IOE-II & 1.65 & Interferon induced protein with tetratricopeptide repeats I \\
\hline IFI44 & $5.86 \mathrm{E}-16$ & 1.64 & Interferon induced protein 44 \\
\hline GBP4 & I.29E-20 & 1.56 & Guanylate binding protein 4 \\
\hline TIMMIO & $5.12 \mathrm{E}-\mathrm{II}$ & 1.54 & Translocase of inner mitochondrial membrane 10 \\
\hline IFI44L & $3.54 \mathrm{E}-\mathrm{II}$ & 1.53 & interferon induced protein 44 like \\
\hline \multicolumn{4}{|c|}{ Down regulated genes } \\
\hline KLFI2 & I.9IE-II & -1.02 & Kruppel like factor 12 \\
\hline HOOKI & $3.24 \mathrm{E}-\mathrm{I} 5$ & -1.04 & Hook microtubule tethering protein I \\
\hline OSBPLIO & 4.0IE-10 & -1.06 & Oxysterol binding protein like 10 \\
\hline RNA5S9 & $6.98 \mathrm{E}-12$ & -1.06 & RNA, 5 S ribosomal 9 \\
\hline FAMI3A & $7.19 \mathrm{E}-1 \mathrm{I}$ & -1.09 & Family with sequence similarity 13 member $A$ \\
\hline
\end{tabular}

TB lesion tissue. Langhan's macrophages are typical cells of tuberculous granuloma. Microscopically, they were large, rich in cytoplasm, and pink, with several nuclei arranged around the cytoplasm like a horseshoe (Figure 4B). No characteristic tuberculosis granuloma structure is in the group (Figure 4A).

\section{IHC Staining and Analysis}

After IHC staining, it could be seen under high magnification that IFIT1 had an obvious positive expression in CNS-TB and PTB lesions, especially strong brown positive expression around multiple macrophages (Figure 4D and G), but no obvious positive expression in the control group (Figure $4 \mathrm{C}$ and F). The results of IHC mapping are quantified and processed by Image-Pro Plus software, and then were drawn by GraphPad Prism for scatter plots and statistical analysis. The results are shown in Figure $4 \mathrm{E}$ and $\mathrm{H}$.

\section{Discussion}

Tuberculosis (TB) is still regarded as one of the serious infectious diseases, threatening human life and health. Although CNS-TB accounts for only $1 \%$ of all $M$. tb related diseases, it is closely related to increasing morbidity and mortality. ${ }^{10,21}$ When $M$. to infects the central nervous system, CNS-TB mainly manifests as tuberculous meningitis (TBM) and intracranial tuberculous mass lesion (TBML). ${ }^{22}$ The infection site is different, which makes the clinical manifestation lack specificity. When the basal 
A

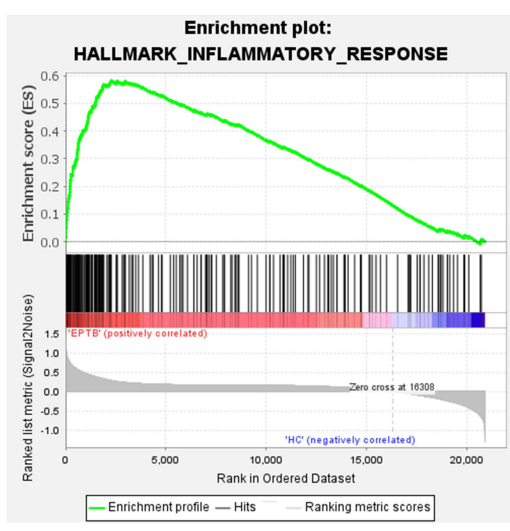

D Enrichment plot: HALLMARK_ALLOGRAFT_REJECTION

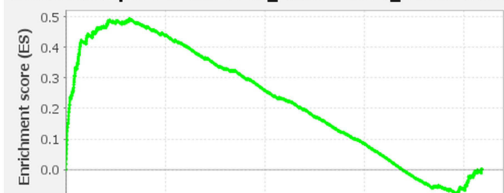

\.0.1

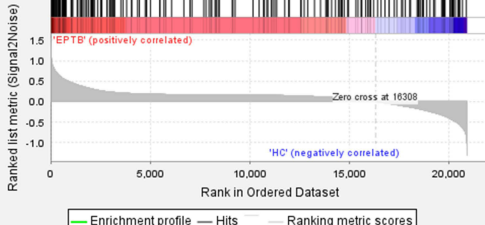

B

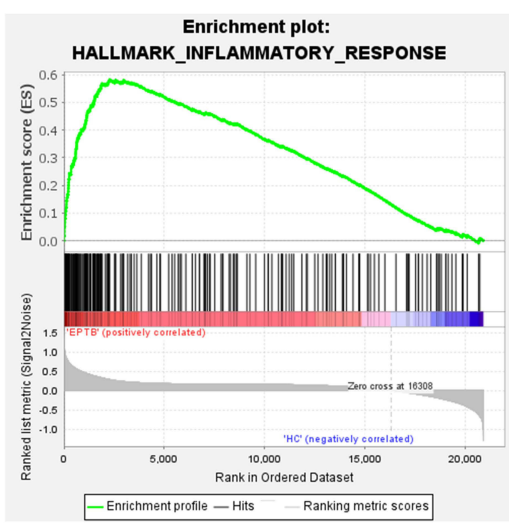

E

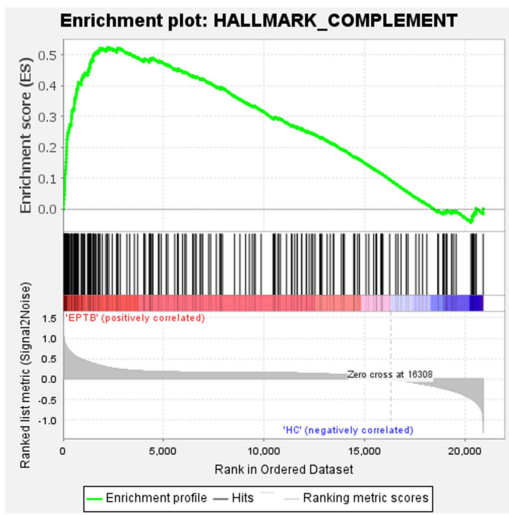

C

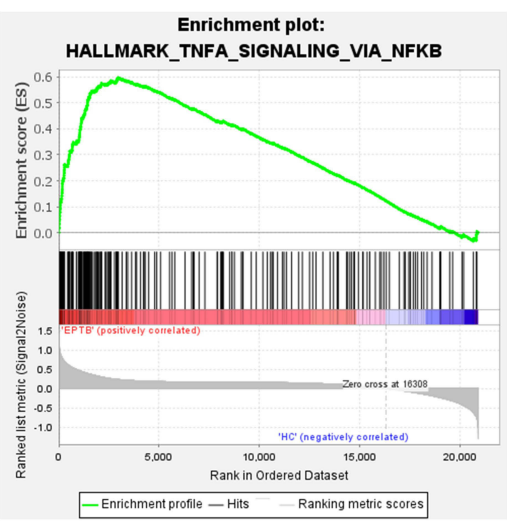

$\mathbf{F}$

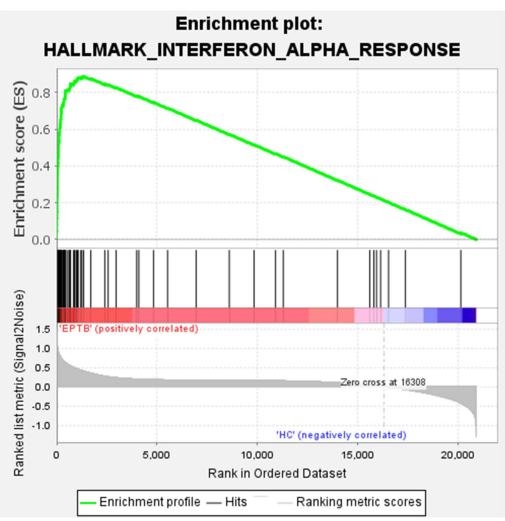

Figure 2 Gene Set Enrichment Analysis enrichment analysis results. (A) GSEA inflammatory signaling pathway. (B) GSEA interferon-gamma signaling pathway. (C) GSEA NF- $K B$ mediated TNFA signaling pathway. (D) GSEA allograft rejection signaling pathway. (E) GSEA complement signaling pathway. (F) GSEA interferon-alpha signaling pathway.

meninges are involved, it will cause local vasculitis and lead to cranial nerve injury, patients have focal neurological dysfunction and cerebral parenchyma ischemia symptoms; when cerebrospinal fluid is blocked by tuberculous brain abscess, patients not only have typical clinical symptoms of increased intracranial pressure, but it also leads to the formation of hydrocephalus. CNS-TB caused cerebral ischemia and hydrocephalus, which can cause serious irreversible complications, this is the main reason for the clinical cause of death. ${ }^{7,21}$ In fact, tuberculosis is associated with many mental/neurological complications and sequelae, and most of the neurological sequelae of CNS-TB are considered to be due to excessive host inflammation. ${ }^{23,24}$ Therefore, inflammatory factors represented by tumor necrosis factor $\alpha$ (TNF- $\alpha$ ) and interferon $\gamma$ (IFN- $\gamma$ ) have received extensive attention from scholars.

In this study, we screened 101 differential genes between EPTB and $\mathrm{HC}, 91$ were up-regulated genes and 10 were down-regulated genes (Figure 1C, Table 1). Metascape online website and GESA software were used

Table 2 GSEA Enrichment Analysis Results

\begin{tabular}{|l|l|l|l|}
\hline GS Follow Link to MSigDB & NES & NOM p-val & FDR q-val \\
\hline HALLMARK_INFLAMMATORY_RESPONSE & 2.19 & 0.004 & 0.024 \\
HALLMARK_INTERFERON_GAMMA_RESPONSE & 2.14 & 0.000 & 0.019 \\
HALLMARK_TNFA_SIGNALING_VIA_NFKB & 2.13 & 0.004 & 0.014 \\
HALLMARK_ALLOGRAFT_REJECTION & 2.10 & 0.004 & 0.012 \\
HALLMARK_COMPLEMENT & 2.06 & 0.008 & 0.016 \\
HALLMARK_INTERFERON_ALPHA_RESPONSE & 2.04 & 0.000 & 0.016 \\
\hline
\end{tabular}




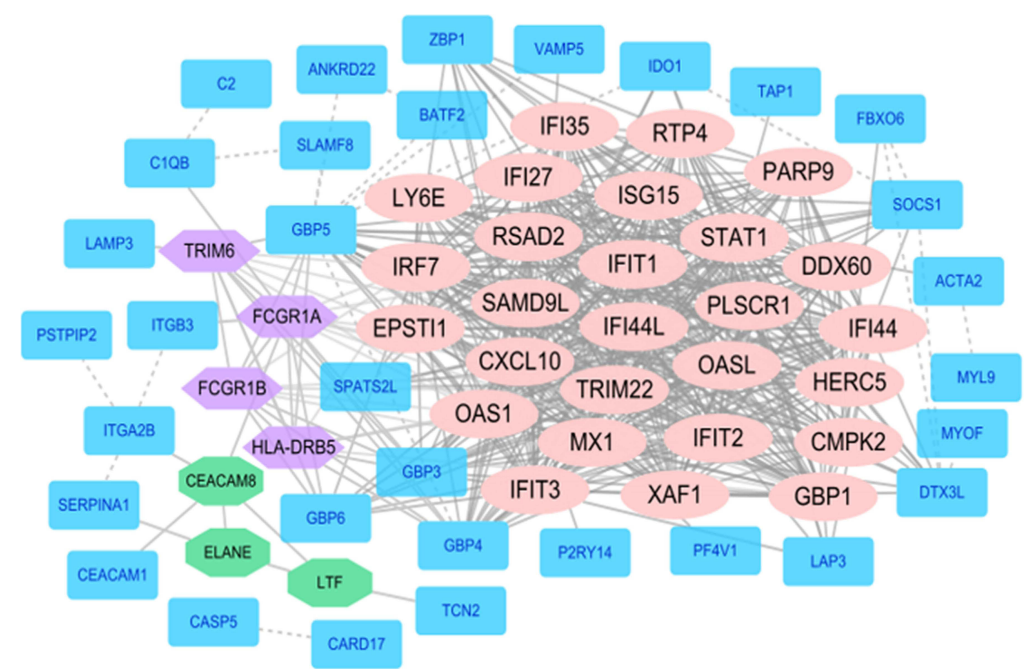

A

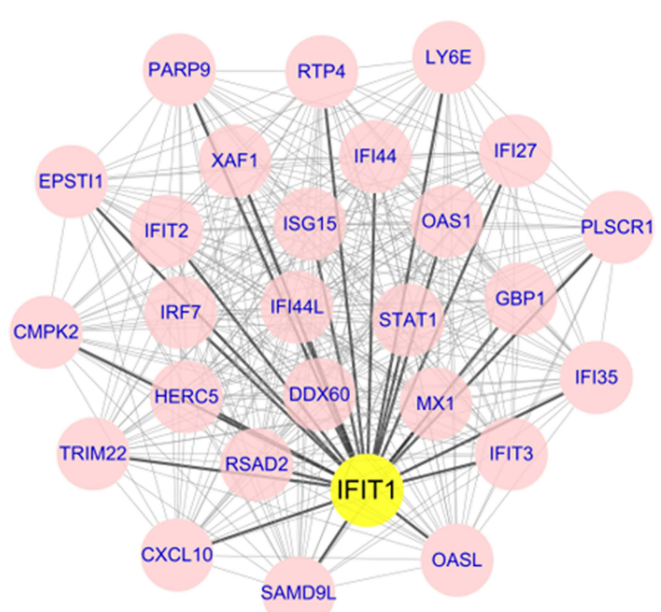

B

Figure 3 The protein-protein interaction network diagrams of differential genes and key genes were visualized by Cytoscape software. (A) A protein-protein interaction network composed of 66 nodes and 509 edges and 3 main gene clusters. (B) Hub gene related protein-protein interaction network diagram.

for enrichment analysis, extrapulmonary tuberculosis mainly activates the body's inflammatory response, and participates in the signaling pathways mediated by cytokines, such as IFN- $\gamma$ and TNF- $\alpha$ (Figures 1D and 2, Table 2). Subsequently, SPRING online and Cytoscape software were used to construct a PPI network map of differential genes, 34 key genes were screened through the MCODE app (Figure 3, Table 3), The most important thing is that we have conducted tissue-specific analysis through BioGPS online, interferon induced protein with tetratricopeptide repeats 1 (IFIT1) was screened out, it was relatively highly expressed in the central nervous system. Subsequently, we collected pathological sections of the lesion tissue of PTB patients, and performed IHC staining. IFIT1 was very positively expressed in the lesion tissue (Figure 4E and F), so IFIT1 was involved in the process of tuberculosis granuloma to some extent. We collected the lesion tissues of 6 CNS-TB patients. These patients

Table 3 MCODE Was Used to Process Data Downloaded from STRING, Specific Data of Gene Cluster

\begin{tabular}{|l|l|l|l|l|}
\hline Cluster & Score & Nodes & Edges & Node IDs \\
\hline I & 25.462 & 27 & 331 & $\begin{array}{l}\text { IFI27, ISGI5, IFI44L, IFI44, RTP4, PARP9, HERC5, SAMD9L, XAFI, GBPI, OASI, CMPK2, IFI35, TRIM22, } \\
\text { LY6E, RSAD2, CXCLI0, MXI, IFIT3, IFITI, STATI, OASL, DDX60, EPSTII, IFIT2, IRF7, PLSCRI }\end{array}$ \\
\hline 2 & 4 & 4 & 6 & HLA-DRB5, FCGRIB, TRIM6, FCGRIA \\
\hline 3 & 3 & 3 & 3 & CEACAM8, ELANE, LTF \\
\hline
\end{tabular}

Table 4 Tissue-Specific Expressed Genes Identified by BioGPS

\begin{tabular}{|l|l|}
\hline System & Genes \\
\hline $\begin{array}{l}\text { Hematologic/immune } \\
\text { system }\end{array}$ & $\begin{array}{l}\text { ISGI5, IFI44, IFI44L, RTP4, PARP9, XAFI, GBPI, OASI, CMPK2, IFI35, TRIM22, CXCLI0, IFIT3, STATI, OASL, DDX60, } \\
\text { IFIT2, IRF7, PLSCRI, HLA-DRB5, FCGRIB, TRIM6, FCGRIA, CEACAM8, ELANE, EPSTII, SAMD9L, MXI, RSAD2 }\end{array}$ \\
\hline Respiratory system & IFI27, LY6E, LTF \\
\hline Central Nervous System & IFITI \\
\hline Genitourinary system & HERC5 \\
\hline
\end{tabular}


Table 5 Clinical Data of CNS-TB Patients

\begin{tabular}{|c|c|c|c|c|c|}
\hline Age & Gender & Nation & The Main Symptoms & $\begin{array}{l}\text { Pathological } \\
\text { Diagnosis }\end{array}$ & Supplement \\
\hline 55 & Male & $\begin{array}{l}\text { Han } \\
\text { nationality }\end{array}$ & frontoparietal mass for 20 days & $\begin{array}{l}\text { Bilateral frontal brain } \\
\text { regions Tuberculous } \\
\text { granuloma }\end{array}$ & $\begin{array}{l}5 \mathrm{~cm} * 5 \mathrm{~cm} \text { subcutaneous nodule on the frontoparietal, } \\
\text { skin-colored, soft, no tenderness, poor mobility, and } \\
\text { fluctuating }\end{array}$ \\
\hline 27 & Male & Minority & $\begin{array}{l}\text { Repeated fever for more than } \\
3 \text { months with paroxysmal } \\
\text { convulsions } 3 \text { times }\end{array}$ & $\begin{array}{l}\text { The right forehead } \\
\text { and parietal lobe } \\
\text { Tuberculous } \\
\text { granuloma }\end{array}$ & $\begin{array}{l}\text { The patient fell repeatedly } 4 \text { times with loss of } \\
\text { consciousness in the past } 15 \text { days. Radiographic } \\
\text { results: intracranial multiple space-occupying lesions, } \\
\text { larger lesions in the right forehead and parietal lobe }\end{array}$ \\
\hline 19 & Female & Minority & $\begin{array}{l}\text { Intermittent headache for } \\
\text { more than } 2 \text { months with the } \\
\text { right limb convulsed } 3 \text { times }\end{array}$ & $\begin{array}{l}\text { Intracranial multiple } \\
\text { caseous necrotizing } \\
\text { Tuberculosis }\end{array}$ & $\begin{array}{l}\text { Radiographic results:Multiple intracranial lesions in the } \\
\text { right cerebellum, left occipital lobe and parietal lobe }\end{array}$ \\
\hline 55 & Male & Minority & $\begin{array}{l}\text { Intermittent headache with left } \\
\text { limb weakness for more than } 5 \\
\text { months }\end{array}$ & $\begin{array}{l}\text { Right temporal } \\
\text { Tuberculous } \\
\text { granuloma }\end{array}$ & $\begin{array}{l}\text { The patient felt impaired vision on the right side. } \\
\text { Radiographic results: edema on the right temporal } \\
\text { side, granulomatous inflammation with massive } \\
\text { necrosis }\end{array}$ \\
\hline 81 & Male & Minority & $\begin{array}{l}\text { Intermittent headache with } \\
\text { vision loss for } 3 \text { weeks }\end{array}$ & $\begin{array}{l}\text { Left frontotemporal } \\
\text { region Tuberculous } \\
\text { granuloma }\end{array}$ & $\begin{array}{c}\text { The patient had dizziness, headache and vomiting I } \\
\text { time. Radiographic results: space-occupying lesions in } \\
\text { the left frontotemporal area }\end{array}$ \\
\hline $4 I$ & Male & Minority & $\begin{array}{l}\text { Intermittent headache with } \\
\text { vomiting for } 2 \text { weeks }\end{array}$ & $\begin{array}{l}\text { Cerebellar } \\
\text { Tuberculous } \\
\text { granuloma }\end{array}$ & $\begin{array}{l}\text { The patient developed unsteady sitting and walking } 7 \\
\text { days ago. Imaging results: cerebellar space-occupying } \\
\text { lesions, supratentorial ventricles with dilatation and } \\
\text { hydrocephalus }\end{array}$ \\
\hline
\end{tabular}

presented to the hospital with symptoms such as dizziness, headache, nausea and vomiting, after admission the hospital performed radiographic examinations, pathological examinations, or pathogenic examinations, which indicated tuberculosis lesions. After surgery, we collected the patient's pathological wax block of lesion tissue and distal part, performed IHC staining. The IHC results showed that IFIT1 was highly expressed in the lesion tissue and statistically significant. Therefore, we believe that IFIT1 plays a role in the course of CNS-TB.

Interferon-stimulating genes (ISGs) is a kind of gene induced by IFN and plays an important role in the body. The interferon induced protein with tetratricopeptide repeats (IFIT) protein family, exists in the cytoplasm, actively participates in the process of anti-microbial infection and inhibits malignant cells. ${ }^{25,26}$ The human genome encodes five sequences (IFIT1, IFIT2, IFIT3, IFIT5 and IFIT1B), IFIT1 was first discovered, ${ }^{27}$ it is also known as ISG56, located on chromosome 10, mainly induced and secreted by IFN- $\alpha$ and IFN- $\gamma{ }^{28}$ Because IFIT1 contains a tetrapeptide repetitive (TPR) domain composed of 34 amino acids, forming a helix-corner-helix structure, making it easy to bind with various proteins, thus interacting with each other, and participating in a variety of biological processes, such as signal transduction, cell proliferation and apoptosis. ${ }^{27,29} \mathrm{In}$ our study, bioinformatics analysis screened out IFIT1 as an important gene involved in EPTB, and its relatively high expression in the central nervous system (Table 4), we realized that it might be a potential molecule involved in the course of CNS-TB. We performed IHC staining in the lesion tissues of PTB and CNS-TB, IFIT1 was highly expressed in the multinucleated macrophages aggregated sites (Figure $4 \mathrm{C}-\mathrm{H}, \mathrm{p}<0.05$ ). Therefore, we have reason to think, IFIT1 is involved in the process of tuberculous granuloma. Although IFIT1 is not specifically expressed in the central nervous system, we have noticed that, in CNS-TB, IFIT1 is significantly higher than PTB (Figure 4D vs Figure 4G). Therefore, we believe that IFIT1 plays a role when M. tb invades the central nervous system.

For a long time, IFIT1 has been considered as a cytokine, it is closely related to innate immunity, and its important role in antiviral immunity has been widely studied. ${ }^{30,31}$ In recent 

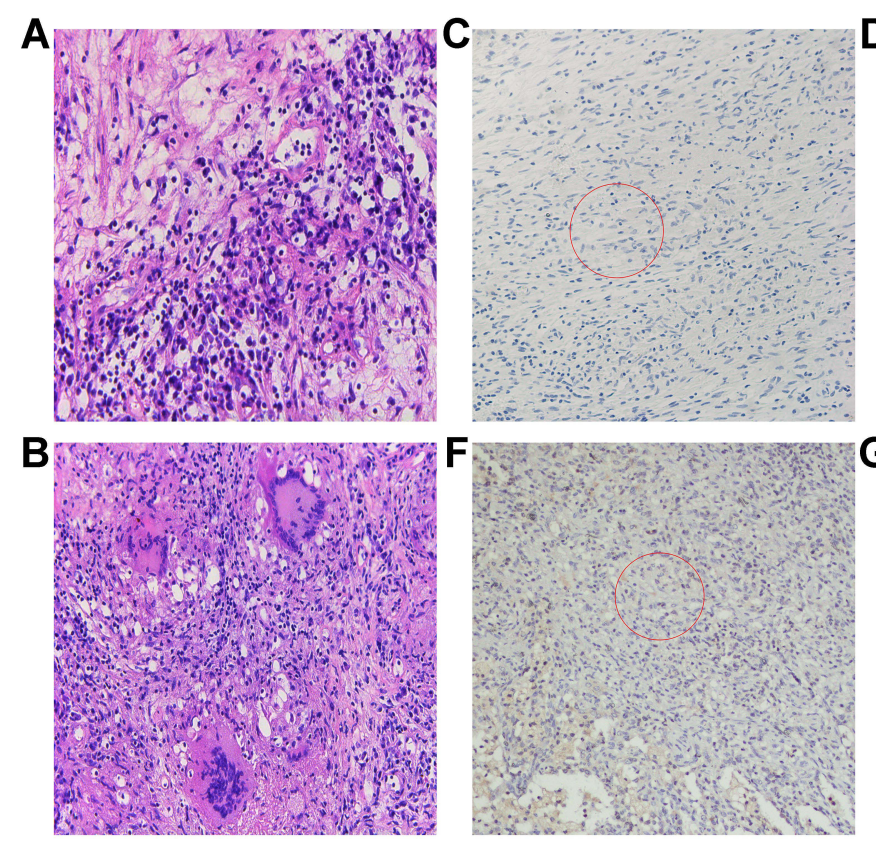
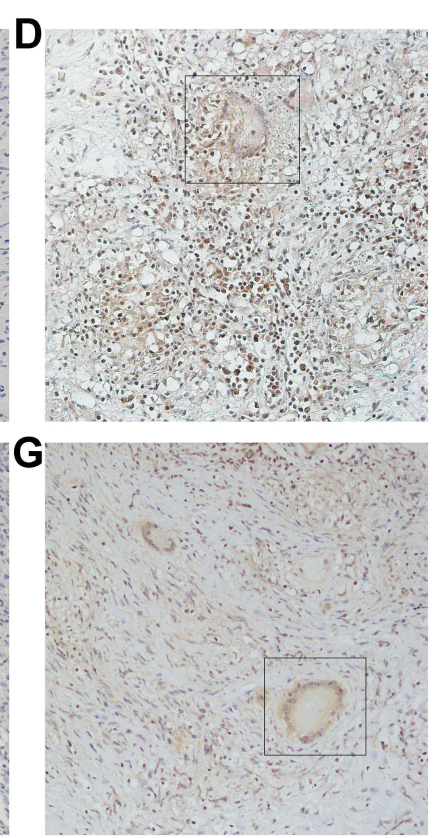
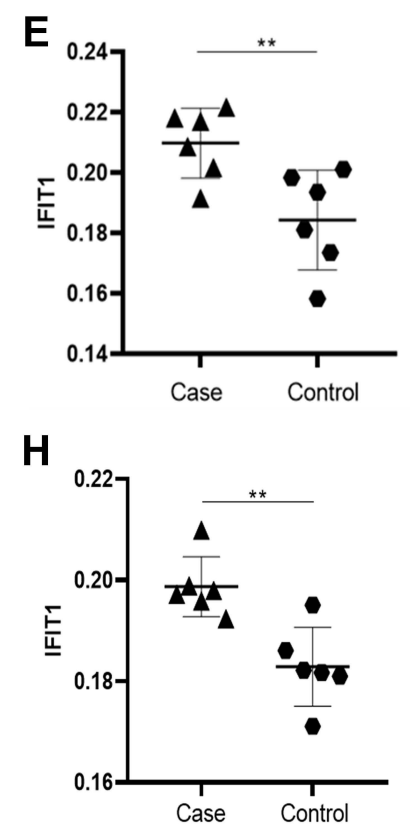

Figure 4 Hematoxylin and Eosin staining and IFITI typical IHC staining results and analysis. (A and B) Central nervous system tuberculosis hematoxylin and eosin (H\&E) staining of lesion tissue and control tissue. (C-E) Central nervous system tuberculosis IFITI typical Immunohistochemistry staining. (F-H) Tuberculosis IFITI typical Immunohistochemistry staining. ${ }^{* *} \mathrm{p}<0.05$, The difference was statistically significant.

years, IFIT1 participation in host cell anti-pathogen infections has gradually attracted attention, but the inflammatory response caused by M. tb infection needs to be supplemented. In this study, DEGs was mainly concentrated in the inflammatory response pathway and IFN-mediated signal pathway. GSEA enrichment analysis showed that IFIT1 made an important contribution to the IFN- $\gamma$ mediated signal pathway (RUNNING ES $=0.46$ ). Therefore, IFIT1 is very likely to participate in the course of CNS-TB through the IFN- $\gamma$ mediated signal pathway In fact, IFIT1 can inhibit the expression of many inflammatory factors through a variety of signal pathways, such as NF-KB and MAPK. In addition, it can also play a role in limiting bacterial escape from internal lysosomes through the IFN pathway. ${ }^{32-34}$ We note that, in central nervous system diseases IFIT1's biological role has been verified, but it has not been paid attention to in CNS-TB. In the occurrence and development of TB disease, IFIT1 may play an important biological role by relying on IFN- $\gamma$, through bioinformation analysis, and IFIT1 expression in the lesions of CNS-TB and PTB patients was verified by immunohistochemistry. To provide new ideas for further exploration of the pathogenesis of CNS-TB in the future.

With regard to the etiology of CNS-TB, it is currently believed to be caused by the expansion of the infected lesions in the shell focal lesions adjacent to the cortex to the subarachnoid space, and it is also believed to be caused by the meninges or cortex infected by M. tb caused by hematogenous spread. ${ }^{35}$ TBM is the most common, characterized by clinical symptoms such as fever, vomiting and apathy, and cranial nerve dysfunction. Fever, headache, epilepsy, focal dysfunction and papillary edema may occur in patients with TBML, depending on the site of the disease. ${ }^{10,35}$ Even if these clinical manifestations can be relieved, they may easily leave neurological/mental sequelae. In particular, the complications such as cerebral ischemia and hydrocephalus caused by CNS-TB are irreversible, these will cause great misfortune to the patients and their families. In our study, IFIT1, a potential molecule, was found to be expressed in the lesions of CNS-TB. Meanwhile, studies have shown that IFIT1 is promising as a marker for detecting potential TB treatment, ${ }^{36}$ Therefore, we hope that the results of this study, can make new thinking for more scholars to further explore the pathogenesis of the disease, and provide new ideas for future research and development of CNS-TB treatment. We firmly believe this will be important for improving the patient's and the patient's family's life quality.

\section{Data Sharing Statement}

The data used to support the findings of this study are included within the article.

\section{Acknowledgments}

This work was supported by the National Natural Science Foundation of China $(82060609 ; 81660353)$ the State Key 
Laboratory of Pathogenesis, Prevention and Treatment of High Incidence Diseases in Central Asia (SKL-HIDCA2017-12; SKL-HIDCA-2020-38; SKL-HIDCA-2020-37; SKL-HIDCA-2020-35; SKL-HIDCA-2020-36) and the Major scientific and technological projects in Xinjiang Uygur Autonomous Region (2017A03006-2) and project supported by Hainan Province Clinical Medical Center.

\section{Author Contributions}

Wenbin Qiao, Jiahui Fan and Xiaoqian Shang contributed equally to the study. All authors contributed to data analysis, drafting or revising the article, gave final approval of the version to be published, agreed to the submitted journal, and agree to be accountable for all aspects of the work.

\section{Disclosure}

The authors have declared that no competing interest exists.

\section{References}

1. World Health Organization. Global tuberculosis report 2020. World Health Organization; 2020. Available from: https://apps.who.int/iris/ handle/10665/336069. Accessed November 18, 2021. License: CC BY-NC-SA 3.0 IGO.

2. Hayward S, Rustage K, Nellums L, et al. Extrapulmonary tuberculosis among migrants in Europe, 1995 to 2017. J Clin Microbiol Infect. 2020;27(9):1347-e1.

3. Pang Y, An J, Shu W, et al. Epidemiology of extrapulmonary tuberculosis among inpatients, China, 2008-2017. Emerg Infect Dis. 2019;25(3):457-464. doi:10.3201/eid2503.180572

4. Wingfield T, MacPherson P, Cleary P, Ormerod L. High prevalence of TB disease in contacts of adults with extrapulmonary TB. $J$ Thorax. 2018;73(8):785-787. doi:10.1136/thoraxjnl-2017-210202

5. Humphreys A, Abbara A, Williams S, et al. Screening contacts of patients with extrapulmonary TB for latent TB infection. Thorax. 2018;73(3):277-278. doi:10.1136/thoraxjnl-2016-209639

6. Kerkhoff AD, Sossen B, Schutz C, et al. Diagnostic sensitivity of SILVAMP TB-LAM (FujiLAM) point-of-care urine assay for extra-pulmonary tuberculosis in people living with HIV. Eur Respir J. 2020;55(2):1901259. doi:10.1183/13993003.01259-2019

7. Bomanji J, Sharma R, Mittal BR, et al. PET/CT features of extrapulmonary tuberculosis at first clinical presentation: a cross-sectional observational 18F-FDG imaging study across six countries. Eur Respir J. 2020;55(2):1901959. doi:10.1183/13993003.01959-2019

8. Venkatraman N, King T, Bell D, et al. High levels of neurological involvement but low mortality in miliary tuberculosis: a 6-year case-series from the UK. Eur Respir J. 2016;47(5):1578-1581. doi:10.1183/13993003.01958-2015

9. Leonard J. Central nervous system tuberculosis. J Microbiol Spectr. 2017;5(2):5-2.

10. Kilicoglu G, Ozturk-Engin D, Tireli H, et al. Radiological data in microbiologically confirmed central nervous system tuberculosis. Clin Microbiol Infect. 2018;24(10):1109-1110. doi:10.1016/j. cmi.2018.05.024

11. Rock RB, Olin M, Baker CA, Molitor TW, Peterson PK. Central nervous system tuberculosis: pathogenesis and clinical aspects. Clin Microbiol Rev. 2008;21(2):243-261. doi:10.1128/CMR.00042-07
12. Piermattei A, Migliara G, Di Sante G, et al. Toll-like receptor 2 mediates in vivo pro- and anti-inflammatory effects of mycobacterium tuberculosis and modulates autoimmune encephalomyelitis. Front Immunol. 2016;7:191. doi:10.3389/fimmu.2016.00191

13. O'Connor RA, Li X, Blumerman S, Anderton SM, Noelle RJ, Dalton DK. Adjuvant immunotherapy of experimental autoimmune encephalomyelitis: immature myeloid cells expressing CXCL10 and CXCL16 Attract CXCR3+CXCR6+ and myelin-specific T cells to the draining lymph nodes rather than the central nervous system. J Immunol. 2012;188(5):2093-2101. doi:10.4049/jimmunol.1101118

14. Hsu N-J, Francisco NM, Keeton R, et al. Myeloid and T cell-derived TNF protects against central nervous system tuberculosis. Front Immunol. 2017;8. doi:10.3389/fimmu.2017.00180

15. Blankley S, Graham CM, Turner J, et al. The transcriptional signature of active tuberculosis reflects symptom status in extra-pulmonary and pulmonary tuberculosis. PLoS One. 2016;11(10):e0162220. doi:10.1371/journal.pone.0162220

16. Ritchie M, Phipson B, Wu D, et al. limma powers differential expression analyses for RNA-sequencing and microarray studies. $J$ Nucleic Acids Res. 2015;43(7):e47. doi:10.1093/nar/gkv007

17. Zhou Y, Zhou B, Pache L, et al. Metascape provides a biologist-oriented resource for the analysis of systems-level datasets. J Nature Commun. 2019;10(1):1523. doi:10.1038/s41467019-09234-6

18. Szklarczyk D, Morris J, Cook H, et al. The STRING database in 2017: quality-controlled protein-protein association networks, made broadly accessible. J Nucleic Acids Res. 2017;45(D1):D362-D368. doi:10.1093/nar/gkw937

19. Reimand J, Isserlin R, Voisin V, et al. Pathway enrichment analysis and visualization of omics data using g: profiler, GSEA, Cytoscape and enrichmentmap. J Nat Protoc. 2019;14(2):482-517. doi:10.1038/ s41596-018-0103-9

20. Wang $\mathrm{H}$, Zhu $\mathrm{H}$, Zhu W, et al. Bioinformatic analysis identifies potential key genes in the pathogenesis of turner syndrome. Front Endocrinol. 2020;11:104.

21. Jain A, Goyal MK, Mittal BR, et al. 18FDG-PET is sensitive tool for detection of extracranial tuberculous foci in central nervous system tuberculosis - preliminary observations from a tertiary care center in northern India. J Neurol Sci. 2020;409:116585.

22. Logan C, Mullender C, Mirfenderesky M, et al. Presentations and outcomes of central nervous system TB in a UK cohort: the high burden of neurological morbidity. $J$ Infect. 2020;82(1):90-97.

23. Robertson KR, Oladeji B, Jiang H, et al. Human immunodeficiency virus type 1 and tuberculosis coinfection in multinational, resource-limited settings: increased neurological dysfunction. Clin Infect Dis. 2019;68(10):1739-1746. doi:10.1093/cid/ciy718

24. van Toorn R, Solomons R, Seddon J, Schoeman J. Thalidomide use for complicated central nervous system tuberculosis in children: insights from an observational cohort. J Clin Infect Dis. 2020;72(5): e136-e145.

25. Fleith R, Mears H, Leong X, et al. IFIT3 and IFIT2/3 promote IFIT1-mediated translation inhibition by enhancing binding to non-self RNA. Nucleic Acids Res. 2018;46(10):5269-5285. doi:10.1093/nar/gky191

26. Katibah G, Lee H, Huizar J, Vogan J, Alber T, Collins KJ. tRNA binding, structure, and localization of the human interferon-induced protein IFIT5. Mol Cell. 2013;49(4):743-750. doi:10.1016/j. molcel.2012.12.015

27. Wathelet M, Moutschen S, Defilippi P, et al. Molecular cloning, full-length sequence and preliminary characterization of a $56-\mathrm{kDa}$ protein induced by human interferons. Eur J Biochem. 1986;155 (1):11-17. doi:10.1111/j.1432-1033.1986.tb09452.x

28. Choi Y, Bowman J, Jung JJI. A talented duo: IFIT1 and IFIT3 patrol viral RNA caps. Immunity. 2018;48(3):474-476. doi:10.1016/j. immuni.2018.03.001 
29. Zhang J, Chen Y, Lin G, et al. High IFIT1 expression predicts improved clinical outcome, and IFIT1 along with MGMT more accurately predicts prognosis in newly diagnosed glioblastoma. Hum Pathol. 2016;52:136-144. doi:10.1016/j.humpath.2016.01.013

30. Zhang L, Wang B, Li L, et al. Antiviral effects of IFIT1 in human cytomegalovirus-infected fetal astrocytes. J Med Virol. 2017;89 (4):672-684. doi:10.1002/jmv.24674

31. Li H, Yang L, Wu C, et al. Expression and prognostic value of IFIT1 and IFITM3 in head and neck squamous cell carcinoma. Am J Clin Pathol. 2020;153(5):618-629. doi:10.1093/ajcp/aqz205

32. John SP, Sun J, Carlson RJ, et al. IFIT1 exerts opposing regulatory effects on the inflammatory and interferon gene programs in LPS-activated human macrophages. Cell Rep. 2018;25(1):95-106. e6. doi:10.1016/j.celrep.2018.09.002

33. Tabellini G, Vairo D, Scomodon O, et al. Impaired natural killer cell functions in patients with signal transducer and activator of transcription 1 (STAT1) gain-of-function mutations. J Allergy Clin Immunol. 2017;140(2):553-564.e4. doi:10.1016/j.jaci.2016.10.051
34. Hare NJ, Chan B, Chan E, Kaufman KL, Britton WJ, Saunders BM. Microparticles released fromMycobacterium tuberculosis-infected human macrophages contain increased levels of the type I interferon inducible proteins including ISG15. Proteomics. 2015;15(17):3020-3029. doi:10.1002/pmic.201400610

35. Zunt JJC. Tuberculosis of the central nervous system. Neuroinfect Dis. 2018;24:1422-1438.

36. de Oyarzabal E, García-García L, Rangel-Escareño C, et al. Expression of USP18 and IL2RA is increased in individuals receiving latent tuberculosis treatment with isoniazid. J Immunol Res. 2019;2019:1297131. doi:10.1155/2019/1297131
Infection and Drug Resistance

\section{Publish your work in this journal}

Infection and Drug Resistance is an international, peer-reviewed openaccess journal that focuses on the optimal treatment of infection (bacterial, fungal and viral) and the development and institution of preventive strategies to minimize the development and spread of resistance. The journal is specifically concerned with the epidemiology of
Dovepress

antibiotic resistance and the mechanisms of resistance development and diffusion in both hospitals and the community. The manuscript management system is completely online and includes a very quick and fair peerreview system, which is all easy to use. Visit http://www.dovepress.com/ testimonials.php to read real quotes from published authors. 